\title{
Point-Based Geometric Deformable Models for Medical Image Segmentation
}

\author{
Hon Pong $\mathrm{Ho}^{1}$, Yunmei Chen ${ }^{2}$, Huafeng Liu ${ }^{1,3}$, and Pengcheng Shi ${ }^{1}$ \\ 1 Dept. of EEE, Hong Kong University of Science \& Technology, Hong Kong \\ 2 Dept. of Mathematics, University of Florida, Gainesville, USA \\ 3 State Key Lab of Modern Optical Instrumentation, Zhejiang University, Hangzhou, China \\ \{garyho@ust.hk, yun@math.ufl.edu, eeliuhf@ust.hk, eeship@ust.hk\}
}

\begin{abstract}
Conventional level set based image segmentations are performed upon certain underlying grid/mesh structures for explicit spatial discretization of the problem and evolution domains. Such computational grids, however, lead to typically expensive and difficult grid refinement/remeshing problems whenever tradeoffs between time and precision are deemed necessary. In this paper, we present the idea of performing level set evolution in a point-based environment where the sampling location and density of the domains are adaptively determined by level set geometry and image information, thus rid of the needs for computational grids and the associated refinements. We have implemented the general geometric deformable models using this representation and computational strategy, including the incorporation of region-based prior information in both domain sampling and curve evolution processes, and have evaluated the performance of the method on synthetic data with ground truth and performed surface segmentation of brain structures from three-dimensional magnetic resonance images.
\end{abstract}

\section{Introduction}

The geometric nature of the geometric deformable models (GDMs) gives them several often desired properties, i.e. independence from parametrization, easy computation of geometrical characteristics such as curvatures, and natural compatibility with topological changes [1,7]. Nevertheless, trivial level set sampling using structured image grid could significantly decrease the computational efficiency when computer memory becomes the bottleneck of the segmentation process, especially for large 3D medical images which have been becoming increasingly available. Fast marching scheme and the narrowband search have sofar provided some reliefs.

However, since most GDMs rely on the finite difference computational schemes, the resolutions of the final results are fundamentally dependent on the computational grids used to solve the discreteized level set partial differential equations (PDEs). Adaptive grid techniques to locally modify the grid structure based on certain criteria is a popular solution. The local refinement methods place additional grid points when (i.e. during narrowband refinement) and where (i.e. high gradient areas) they are needed to avoid expensive global refinement procedure [3]. More recently, moving grid methods have been proposed as simpler alternatives to the local refinement methods [4]. But the local sampling rate for such moving grid strategy is still upper-bounded by the number of initial grid lines used. Regardless of the choices of computational grids, task-specific 
incorporation of image force and prior knowledge also plays essential roles in achieving accurate and attractive segmentation results, especially for medical images [2].

In the Lagrangian formulation of parametric deformable models (PDMs) and motion tracking, meshfree particle methods have been introduced as more efficient and possibly more effective object representation and computation alternatives because of their trivial $h-p$ adaptivity [6]. In addition, it has been shown that, for arbitrary shape modeling, point-sampled representation allows user-defined large deformations on the object shape [10]. Following the same spirit, it is easy to see that if general level set PDE computation can be conducted in a point-based environment for both shape and evolution (deformation) domains, we can almost freely adjust the sampling rate of the domains without many extra efforts. This can be achieved through point-wise continuous approximations of the domain and of the level set functions through polynomials fitting using local point cloud. With such inspiration, we have introduced a point-based numerical scheme for domain representation and computation [5].

In this paper, we extend the numerical scheme of [5] to point-based geometric deformable models (GDMs) for medical image segmentation. In particular, through adaptive sampling points distribution and level set formulation, our curve evolution formulation incorporates region-based prior information, which is especially useful for brain segmentation because the intensity homogeneity of the brain structures can compensate noisy or confusing boundary information. Spatial derivatives of the point-sampled level set function are obtained through moving least squares (MLS) approximation. Because of the $h-p$ adaptive nature of the sampling points, our strategy naturally possesses multiscale property and performs fast marching and local refinement concurrently.

\section{Methodology}

\subsection{Geometric Deformable Models}

Level Set Formulation: Suppose we want to model a dynamic deformable contour $C(t) \in \mathbb{R}^{d}$ under a known velocity field $F(\mathbf{x}, C(t))$, where $\mathbf{x} \in \mathbb{R}^{d}$ and the initial contour $C(0)$ is given. The dynamic equation for the moving contour is then:

$$
\frac{d C(t)}{d t}=F(\mathbf{x}, C(t))
$$

where $F$ is known as the speed term that determines the particular way under which $C(t)$ deforms. If we want $C(t)$ to stop at certain locations in space, such as object boundary in image segmentation cases, then those locations should afford small or zero $F(\mathbf{x})$ values. If the targeted object is complex or the image data is noisy, the actual $F$ field could be very complicated and the resulted $\left.C(t)\right|_{t \rightarrow \infty}$ requires flexible and high precision representation. Therefore, changing the representation of $C(t)$ into implicit forms had been developed, known as the level set methods [8].

The basic idea of level set methods is to implicitly represent the moving surface $C(t)$ by the zero level set of a higher dimensional hypersurface $\phi$ (the level set function), i.e. the signed distance transformation of $C(t)$ in $\mathbb{R}^{d}$, such that $C(t)=\{\mathbf{x} \mid \phi(\mathbf{x}, t)=$ $\left.0 \forall \mathbf{x} \in \mathbb{R}^{d}\right\}$. The curve evolution is then driven by $F$ in normal directions and we solve for convergence of the dynamic equation by time-domain finite difference: 


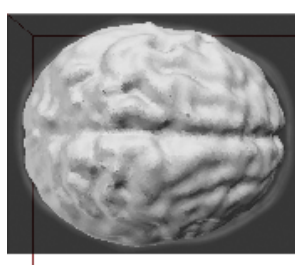

$k=20, F=F_{1}$

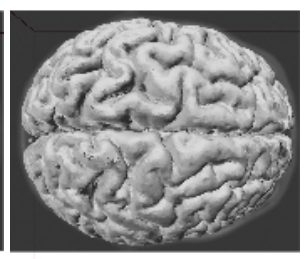

$k=20, F=F_{2}$

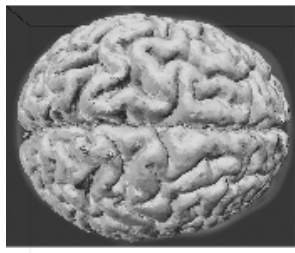

$k=12, F=F_{2}$

Fig. 1. Brain segmentations using same set of sampling point cloud with different forces in Eqn. (2) and samples per influence domain $(k)$. The $1^{\text {st }}$ figure was segmented without region-based prior model while the $2^{\text {nd }}$ and $3^{\text {rd }}$ are significantly improved results by using both boundary image information and regional priors. In the rightmost two figures, larger $k$ makes the surface smoother while smaller $k$ reduces computation but is more noise sensitive.

$$
\phi^{t+\Delta t}=\phi^{t}+\Delta t F|\nabla \phi|
$$

Construction of the Speed Term $\boldsymbol{F}$ : To achieve desirable segmentation results, taskspecific incorporation of image force and other knowledge for the speed term $F$ plays essential roles [7,9]. One of the most popular $F$ formulations states:

$$
F_{1}=\beta g \kappa-(1-\beta) g \hat{v} \cdot \frac{\nabla \phi}{|\nabla \phi|}
$$

where $g=\frac{1}{1+|\nabla G * I|}$ is the image gradient force, $\kappa$ is the contour curvature, $\hat{v}$ is the gradient vector flow (GVF), $\beta \in[0,1]$ is a weighting parameter, and $G$ denotes the gaussian smoothing kernel. This $F$ form, however, may not produce appropriate segmentation results. In Fig 1, the less detailed surface in the leftmost figure was segmented using $F_{1}$. An initial guess surface was first placed inside the brain structure and then expanded outward driven. When the surface encountered sharp edges, the GVF force became dominant and attracted that portions of surface towards their nearest sharp edges. However, the image intensity gradient at certain areas of the brain image is too weak to slow down the surface expansion. Further, GVF itself is a diffusion process such that weak edges would be covered by the impact of nearby sharp edges. In the brain image, the skull layer actually has sharper boundaries than the brain surface, and thus would make the whole surface move outward beyond its proper location.

To address this problem, we further constrain the surface expansion by region-based information so that the surface would expand only if it is likely to be inside our target. The likelihood is defined by the similarity between local image intensities and a statistical mean intensity $I_{m}$ with variance $\sigma^{2}$, constructed from prior knowledge of the structure. With this additional information, we define our new speed term as:

$$
F_{2}=\alpha g \kappa-\beta g \hat{v} \cdot \frac{\nabla \phi}{|\nabla \phi|}+\gamma\left(F_{a}-e^{-\frac{1}{n \sigma^{2}} \sum\left|I_{\mathbf{x}}-I_{m}\right|}\right)
$$

where $\alpha, \beta$ and $\gamma$ are three weighting constants. We assume our $C(t)$ surface evolves outward. $F_{a}$ is an additional natural expanding speed. To avoid excessive expansion, 
$\alpha$ and $\beta$ should be smaller than the original weighings in $F_{1}$. In the third term of $F_{2}$, we modify the natural speed by prior information: $I_{m} \in[0,1]$ and $\sigma^{2} \in \mathbb{R}$. $I_{\mathbf{x}} \in$ $[0,1]$ are image intensities around point $\mathbf{x}$ and $n$ is the number of neighboring points. Then, $\sum\left|I_{\mathbf{x}}-I_{m}\right| / n$, the average inconsistence of local intensities will slow down the surface $C(t)$ motion. We would like to suggest $F_{a}>1$ such that the whole third term will be positive if $\mathbf{x}$ is inside the target and negative if outside. This would have similar effect as the GVF force in the second term.

\subsection{Numerical Implementation of Point-Based GDMs}

Adaptive Domain Sampling with Data- and Prior-Driven Point Cloud: Rather than using image grid or other mesh structures, we use a set of adaptively distributed points $\mathbf{x}_{i} \in \mathbb{R}^{d}$ to dynamically sample the spatial domains for Eqn. (2) [5]. The position of these sampling points can be flexible and adaptive, and we design to put more points near areas of richer features (i.e. image gradient, geometrical characteristics of the evolving contour, prior knowledge of boundary likelihood from experience or training images, etc.) in order to achieve higher precision for the $\phi$ function. On the other hand, for relatively featureless or uninteresting areas such as the background or homogeneous region inside the object, we only need to maintain a minimal amount of points to support proper $\phi$ evaluation for $C(t)$ contour propagation.

Obtaining Derivatives of $\phi$ : While the unstructured sampling point cloud affords greater adaptivity towards data and priors, the calculation of $\nabla \phi$ is no longer as straightforward as the conventional finite difference implementations because the relationship between the sampling points are no longer directly defined.

Assuming that the $\phi$ function at any location $\mathbf{x}$ is a well-behaved polynomial surface within a small area around $\mathbf{x}$, we can use $\phi\left(\mathbf{x}_{i}\right)$ of the neighboring nodes $\mathbf{x}_{i}$ in that area to recover the coefficients of $\phi$ surface by performing the moving least square (MLS) approximation [6]. Calling such local area the influence domain or support region of $\mathbf{x}$, all nodes inside the influence domain will have certain amount of effects on the recovered coefficients, weighted by their relative distance to $\mathbf{x}$. In our particular implementation, we actually select the proper number of neighboring nodes $k$ for

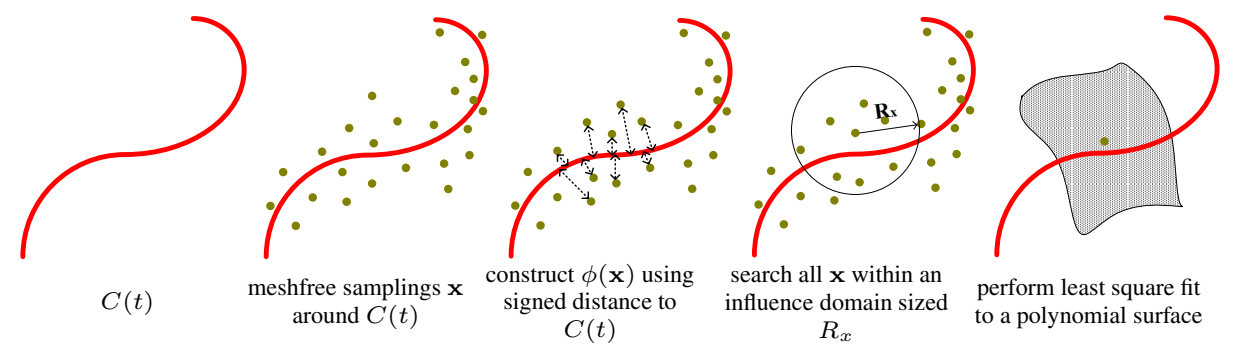

Fig. 2. From left to right: steps to construct $|\nabla \phi|$ on point cloud using moving least square fitting. The fitting is done on the $\phi(\mathbf{x})$ values. Once the polynomial coefficients have been recovered, the derivative of the fitted surface $(\nabla \phi)$ can also be calculated. Then the $\phi$ can be updated by Eqn. (2) and $C(t+\Delta t)$ is obtained by Eqn.77. Finally, we substitute $C(t)$ by $C(t+\Delta t)$ and the process can be repeated iteratively until $C$ converges. 
$\mathbf{x}$ instead of determining the size of influence domain. Since the node distribution is feature-driven, choosing a fixed number of nearest neighboring nodes for $\mathbf{x}$ effectively imply a feature-adaptive determination of the influence domain size. Fig. 2 gives an overview of the use of influence domain in the point-based GDM implementation.

Let $\tilde{\phi}$ be the MLS-approximated $\phi$ with the form: $\tilde{\phi}(\mathbf{x})=\sum_{j}^{m} p_{j}(\mathbf{x}) a_{j}(\mathbf{x}) \equiv$ $\mathbf{p}^{T}(\mathbf{x}) \mathbf{a}(\mathbf{x})$, where $\mathbf{p}$ is the polynomial basis, $m$ is the order of polynomial, and $\mathbf{a}$ is the coefficient vector. To get $\tilde{\phi}(\mathbf{p})$ at arbitrary location $\mathbf{x}$, we determine a through minimizing the weighted approximation error $J$ of the $\phi\left(\mathbf{x}_{i}\right)$ values of the $k$-nearest neighboring nodes $\mathbf{x}_{i}$, defined as the followings:

$$
\begin{aligned}
J & =\sum_{i}^{k} W\left(\frac{\left|\mathbf{x}-\mathbf{x}_{i}\right|}{R_{\mathbf{x}}}\right)\left[\mathbf{p}^{T}\left(\mathbf{x}_{i}\right) \mathbf{a}(\mathbf{x})-\phi\left(\mathbf{x}_{i}\right)\right]^{2} \\
W(\bar{d}) & = \begin{cases}\frac{2}{3}-4 \bar{d}^{2}+4 \bar{d}^{3} & \text { for } \bar{d} \leq \frac{1}{2} \\
\frac{4}{3}-4 \bar{d}+4 \bar{d}^{2}-\frac{4}{3} \bar{d}^{3} & \text { for } \frac{1}{2} \leq \bar{d} \leq 1 \\
0 & \text { for } \bar{d}>1\end{cases}
\end{aligned}
$$

where $W$ is a cubic spline decaying weight function, $\left|\mathbf{x}-\mathbf{x}_{i}\right|$ is the spatial distance between $\mathbf{x}$ and $\mathbf{x}_{i}$, and $T$ denotes vector transpose. The derivative $\nabla \phi$ can then be approximated by the derivative of the recovered polynomial $\tilde{\phi}$.

Detecting Zero Crossings of $\phi$ : In any level set method, $\phi$ is only a medium for evolving $C(t)$ while the ultimate goal is to properly localize the $C(t)$ surface as the zero level set of $\phi$. However, for the point-based GDMs, the detection of the zero level set can no longer be performed using the widely used Marching Cubes algorithm that requires a grid structure. Instead, we opt to detect the zero-crossings of evolved $\phi[8]$ :

$$
\mathbf{z}_{i}=\mathbf{x}_{i}-\phi\left(\mathbf{x}_{i}, t\right) \frac{\nabla \phi\left(\mathbf{x}_{i}, t\right)}{\left|\nabla \phi\left(\mathbf{x}_{i}, t\right)\right|}
$$

where $\mathbf{z}_{i}$ is the nearest $\phi=0$ position from point $\mathbf{x}_{i}$. The equation assumes $\phi$ is a signed distance function of $C(t)$ and $\nabla \phi$ denotes the normal direction towards nearest $C(t)$ position. The set of $\mathbf{z}_{i}$ becomes the discrete representation of $C(t)$.

\section{Experiments}

\subsection{Grid- and Point-Based Implementations: Comparison on Synthetic Images}

A synthetic image with known boundary (Fig 3 ) is used to evaluate the grid- and pointbased implementations. To make fairer comparison, for the point-based GDMs, we distribute sampling points according to the image gradient magnitude only, where the total number of sampling points is roughly one quarter fewer than the grid-based case. In the grid-based implementation, the Marching Cubes algorithm is used to detect the zero-crossings of $\phi$ and the image grid is used as the sampling grid. For both implementations, $F_{1}$ speed term is used, the image gradient $|\nabla I|$ is computed using central difference, and narrow band speedup is adopted [7].

Different levels of Gaussian noises are added to the phantom image, and the performance of the two implementations are tabulated in Fig 4 . For the mean squared errors (MSE), the point-based GDMs are slightly better than the conventional finite difference 

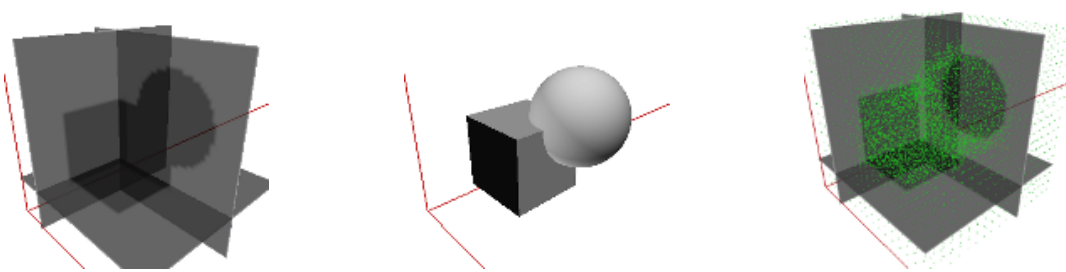

Fig. 3. From left to right: synthetic 3D image, underlying ground truth object, and sampling point cloud for experiments in Fig. 4

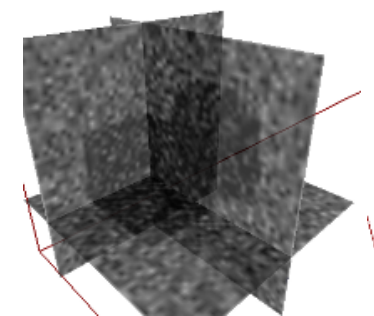

Synthetic image with noise (3dB)

\begin{tabular}{|c|c|c|c|c|c|}
\multicolumn{2}{c}{$(3 \mathrm{~dB})$} & \multicolumn{2}{c}{ grid-based method } & \multicolumn{2}{c|}{ point-based method } \\
\hline & SNR: & $10 \mathrm{~dB}$ & $7 \mathrm{~dB}$ & $5 \mathrm{~dB}$ & $3 \mathrm{~dB}$ \\
\hline \multirow{3}{*}{$\begin{array}{c}\text { Grid-Based } \\
\text { Implementation }\end{array}$} & Time: & $90 \mathrm{sec}$ & $98 \mathrm{sec}$ & $86 \mathrm{sec}$ & $171 \mathrm{sec}$ \\
\cline { 2 - 6 } & MSE: & 0.1619 & 0.1867 & 0.2180 & 0.1970 \\
\cline { 2 - 6 } & Std Deviation: & 0.2410 & 0.2623 & 0.3438 & 0.3222 \\
\cline { 2 - 6 } & Max Error: & 1.3762 & 1.5169 & 1.7272 & 1.9957 \\
\hline \multirow{3}{*}{$\begin{array}{c}\text { Point-Based } \\
\text { Implementation }\end{array}$} & Time: & $100 \mathrm{sec}$ & $84 \mathrm{sec}$ & $102 \mathrm{sec}$ & $100 \mathrm{sec}$ \\
\cline { 2 - 6 } & MSE: & 0.1544 & 0.1892 & 0.2017 & 0.1951 \\
\cline { 2 - 6 } & Std Deviation: & 0.1869 & 0.2185 & 0.2392 & 0.2625 \\
\cline { 2 - 6 } & Max Error: & 1.2911 & 1.6274 & 1.4755 & 1.4260 \\
\hline
\end{tabular}

Fig. 4. Error comparison between point-based and grid-based (finite difference) GDMs on segmentation of 3D synthetic noisy images with known ground truth

GDMs. However, due to the smoothing effect of MLS, they are much more stable in terms of the error standard deviations, especially when the noises are more significant. We also want to point out that, while the MLS procedure is computationally expensive, adaptive point sampling can efficiently limit the total amount of needed points and thus the point-based GDMs are still computationally comparable to the grid-based GDMs for practical usages, as indicating by the processing time.

\subsection{Segmentation of Brain Structures}

In Fig. 11 with uniformly distributed sampling points, we show the effect of the number of points $k$ per influence domain $[1$, or equivalently the sizes of the influence domain. In

\footnotetext{
${ }^{1} k$ is lower bounded to ensure that the MLS system of equations is invertible.
} 

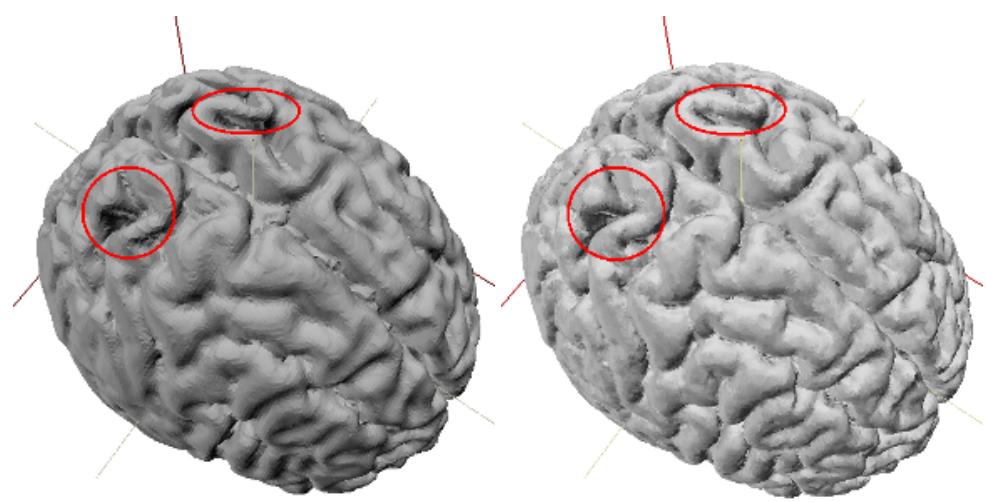

Fig. 5. Comparison between grid-based (left) and point-based (right) brain surface segmentation

general, larger $k$ would produce smoother segmented surface (at higher computational cost), desirable for larger structures and flatter regions. On the other hand, smaller $k$ efficiently yields rougher results, which is actually needed for geometrical details. Our point-based GDMs, however, adopt feature-driven point distribution scheme and thus automatically take care of the selection of $k$ or influence domain size.

Comparison of the point- and grid-based implementations is made for brain surface segmentation (Fig. 5), both using regional priors. We first obtain a coarse segmentation using fewer sampling points which covers the whole image, and then distribute huge amount of points around the coarse segmentation results, with even more points in high image gradient areas. While the difference between the two results is not significant at smooth regions, around extremely thin structures as we have explicitly pointed out, sampling insufficiencies are uncovered by sharp changes of normal directions of the triangles in the grid-based results, extracted by the Marching Cubes algorithm.

Suppose that we have some prior knowledge about the rough locations of certain brain structures, we distribute sampling points around (inside and outside) those areas with higher point density for high probability area. Example of point distribution is

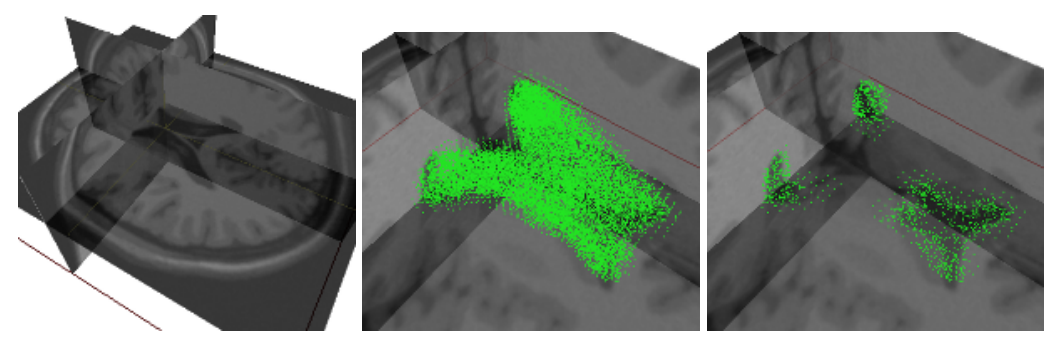

Fig. 6. Left: A Brainweb image (http://www.bic.mni.mcgill.ca/brainweb), $T_{1}$ weighted, $3 \%$ noise. Middle: Sampling nodes are distributed according to prior shape information obtained from training images. These nodes are used to sample the level set function $\phi$ during surface evolution (Fig7). Right: Sampling points on one specific slice of the 3D image. 

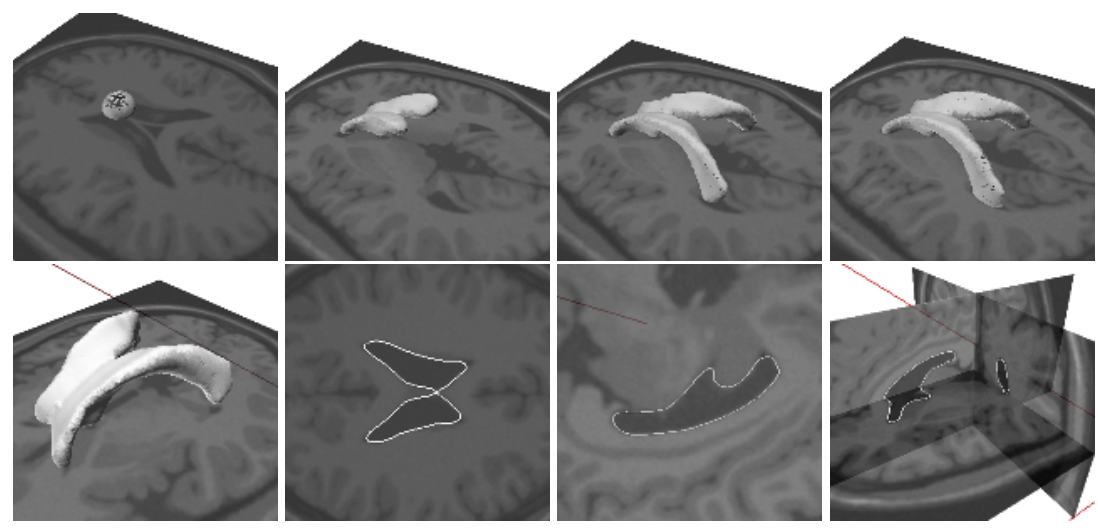

Fig. 7. Top: Brain ventricle segmentation with prior-driven node distribution. Bottom: Segmented brain ventricle surface using point-based GDM, and the intersections of surface with image slices.

shown in Fig. 6 for brain ventricle segmentation, and the point-based GDM evolution process and segmentation results are shown in Fig.7.

\section{Acknowledgement}

This work is supported by NBRPC 2003CB716104, NSFC 60403040, HKUST6252/04E.

\section{References}

1. V. Caselles, R. Kimmel, and G. Sapiro. Geodesic active contours. IJCV, 22:61-79, 1997.

2. Y. Chen, H. Tagare, and et al. Using prior shapes in geometric active contours in a variational framework. International Journal of Computer Vision, 50:315-328, 2002.

3. M. Droske, B. Meyer, M. Rumpf, and C. Schaller. An adaptive level set method for medical image segmentation. In Information Processing in Medical Imaging, pages 416-422, 2001.

4. X. Han, C. Xu, and J. L. Prince. A 2 d moving grid geometric deformable model. In IEEE Conference on Computer Vision and Pattern Recognition, volume 1, pages 153-160, 2003.

5. H.P. Ho, Y. Chen, H. Liu, and P.Shi. Level set active contours on unstructured point cloud. In IEEE Conference on Computer Vision and Pattern Recognition, 2005.

6. H. Liu and P. Shi. Meshfree particle method. In ICCV, pages 289-296, October 2003.

7. R. Malladi, J. A. Sethian, and B. C. Vemuri. Shape modeling with front porpagation: a level set approach. IEEE Trans. on Pattern Analysis and Machine Intelligence, 17:158-175, 1995.

8. S. Osher and R. Fedkiw. Level set methods and dynamic implicit surfaces. Springer-Verlag New York Inc., 2002.

9. N. Paragios, O. Mellina-Gottardo, and V. Ramesh. Gradient vector flow fast geometric active contours. IEEE Trans. on Pattern Analysis and Machine Intelligence, 26(3), March 2004.

10. M. Pauly, R. Keiser, L.P. Kobbelt, and M. Gross. Shape modeling with point-sampled geometry. ACM SIGGRAPH (Trans. Graphics), 22(3):641-650, July 2003. 